\title{
Visual Acuity Inspired Saliency Detection by Using Sparse Features
}

\author{
Yuming Fang ${ }^{\mathrm{a}}$, Weisi Lin ${ }^{\mathrm{b}}$, Zhijun Fang ${ }^{\mathrm{a}, *}$, Zhenzhong Chen ${ }^{\mathrm{c}}$, Chia-Wen \\ Lin $^{\mathrm{d}}$, Chenwei Deng ${ }^{\mathrm{e}}$ \\ ${ }^{a}$ School of Information Technology, Jiangxi University of Finance and Economics, \\ Nanchang 330032, Jiangxi, China \\ ${ }^{b}$ School of Computer Engineering, Nanyang Technological University, 639798 Singapore \\ ${ }^{c}$ School of Remote Sensing and Information Engineering, Wuhan University, Wuhan \\ 430079, China \\ ${ }^{d}$ Department of Electrical Engineering, National Tsing Hua University, Hsinchu 330013, \\ Taiwan \\ ${ }^{e}$ School of Information and Electronics, Beijing Institute of Technology, Beijing 100081, \\ China
}

\section{Abstract}

In this paper, we propose a new computational model of visual attention based on the relevant characteristics of the Human Visual System (HVS) and sparse features. The input image is first divided into small image patches. Then the sparse features of each patch are extracted based on the learned independent components. The human visual acuity is adopted in calculation of the center-surround differences between image patches for saliency extraction. We choose the neighboring patches for center-surround difference calculation based on the relevant characteristics of the HVS. Furthermore, the center-bias factor is adopted to enhance the saliency map. Experimental

\footnotetext{
${ }^{*}$ Corresponding author.

Email addresses: fa0001ng@e.ntu.edu.sg (Yuming Fang), wslin@ntu.edu.sg (Weisi Lin), zjfang@gmail.com (Zhijun Fang), zzchen@whu.edu.cn (Zhenzhong Chen), cwlin@ee.nthu.edu.tw (Chia-Wen Lin), cwdeng@bit.edu.cn (Chenwei Deng)
} 
results show that the proposed saliency detection model achieves better performance than the relevant existing ones on a large public image database with ground truth.

Keywords: Visual Attention, Saliency Detection, Human Visual System, Sparse Features, Human Visual Acuity.

\section{Introduction}

When looking at a visual scene, an observer cannot capture all visual information in the scene with detail at the same time. The visual information in a scene is usually much more than that the human central neural system can process. Selective attention in the Human Visual System (HVS) is an important mechanism to focus on some particular visual information while ignoring the other in visual scenes [39, 43]. There are two types of visual attention mechanisms in the HVS: bottom-up and top-down. Bottom-up attention is a involuntary and task-independent perceptual processing for salient region selection $[16,21,31,36,46]$, while top-down attention is a voluntary perceptual processing influenced by prior knowledge such as tasks

to be performed, feature distribution of targets, etc. [4, 18, 28].

In this paper, we focus on bottom-up visual attention modeling. Currently, bottom-up saliency detection is widely used in various visual processing applications such as retargeting, retrieval, coding, etc. Based on the Feature Integration Theory (FIT) [39], various computational models of visual attention have been proposed to predict human fixation regions $[5,7$, $12,27,42]$. Most of these existing models extract the saliency map of the input image by computing the center-surround differences between image 
patches. However, these models rarely consider other useful characteristics of the HVS. In this study, we propose a acuity-based saliency detection model for images. Sparse features are extracted to represent the image patches for center-surround difference calculation. Additionally, an important characteristic of the HVS, the human acuity, is adopted to weight the center-surround differences between image patches for saliency detection.

Existing studies show that the sparse representation of image statistics exists in the primate visual system. In particular, sparse coding is regarded as an efficient coding strategy in the primary visual cortex $[8,35]$. With such image representation, sparse features of images can be obtained. In this study, we extract sparse features of image patches to compute centersurround differences between image patches.

It has been demonstrated that the HVS is highly space-variant in processing visual information because the retina in the human eye has different densities of cone photoreceptor and ganglion cells [41]. The density of cone receptor and ganglion cells accounts for the visual acuity. The highest density of cone receptors is found in the fovea of retina. With greater retinal eccentricity from the fovea region, the density of the cone receptors becomes lower and thus the visual acuity decreases. We propose to incorporate the characteristics of the HVS in this regard (i.e. the human visual acuity) into the proposed saliency detection model. To be more specific, the human visual acuity is used to weight the center-surround feature differences between image patches for saliency calculation.

In essence, the proposed model first divides the input image into small image patches, whose sparse features are extracted based on the learned basis. 
The center-surround differences between image patches are then calculated by the extracted sparse features. By using the human visual acuity to weight the center-surround differences, we compute the saliency map of the input image. Besides, existing studies demonstrate that the center-bias exists during human fixation and we integrate this factor into the proposed saliency detection model. Different from existing studies which use the Euclidean distance or the Gaussian distribution of Euclidean distance to weight the center-surround differences [7, 42], the proposed model uses a more reasonable weighting method by the human visual acuity for saliency extraction. Additionally, we choose the neighboring image patches for center-surround difference calculation based on the characteristics of the HVS. Experimental results on a large public database with ground truth show the better performance of the proposed saliency detection model over other existing ones.

The reminder of this paper is organized as follows. In Section 2, we introduce the related research work in the research literature. Section 3 describes the proposed model in detail. In Section 4, the experimental results are provided to demonstrate the performance of the proposed model. The final section concludes the paper.

\section{Related Work}

Currently, many computational models of visual attention have been proposed for various multimedia processing applications during the past decades. One of the earliest saliency detection models proposed by Itti et al. is built based on the behavior and the neuronal architecture of the primates' early

visual system [16]. That model computes saliency map by calculating the 
multi-scale center-surround differences from low-level features of intensity, color and orientation. Based on Itti's model, Harel et al. devised a Graphbased Visual Saliency (GBVS) model through utilizing a dissimilarity measure for saliency extraction [11]. Different from Itti's model, GBVS model uses the graph theory to compute the saliency map based on low-level features. Gao et al. calculated the saliency map through a defined centersurround discriminant [5]. The saliency value of each image pixel is computed by the power of a Gabor-like feature set [5]. Goferman et al. designed a context-aware saliency detection model by including more context information in the saliency map [7]. In that study, color information in Lab color space is used for feature extraction [7].

Some studies of visual attention modeling try to calculate the saliency map of the input image in transform domain. Hou et al. built a visual attention model by using the concept of Spectral Residual (SR), and claimed that the SR model can be implemented by log spectra representation of images [12]. Later, Guo et al. found that the phase spectrum is the main factor for the saliency detection in that model [12] and thus designed a phasebased saliency detection model based on Fourier Transform (FT) [10]. In that study, the saliency map is estimated by Inverse Fourier Transform (IFT) on a constant amplitude spectrum and the original phase spectrum [10]. The wavelet coefficients are adopted to build the saliency detection model in [14]. Both local and global contrast are calculated from the wavelet coefficients for saliency estimation [14]. In [34], Murray et al. proposed a saliency detection model by using Inverse Wavelet Transform on the multi-scale features [34]. In that study, the scale-weighting function has been optimized to replicate 
psychophysical data on color perception [34]. Wang et al. proposed a saliency detection model based on the information maximization principle [42]. In that study, they defined the Site Entropy Rate to measure the saliency for images [42].

Recently, there are some spatiotemporal saliency detection models proposed for video sequences. Itti et al. proposed to use a Bayesian model to detect surprising events which attract human attention. In that study, surprising events are calculated by the differences between posterior and prior beliefs for observers [15]. Ma et al. designed a saliency detection model by considering both the top-down and bottom-up mechanisms for video summarization [29]. Zhai et al. proposed a spatiotemporal saliency detection model by fusing spatial and temporal saliency linearly [45]. Le Meur et al. extended their previous saliency detection model from the spatial domain to the spatiotemporal domain [22]. The achromatic, chromatic and temporal features are used to estimate the saliency map of video frames. Li et al. proposed spatiotemporal saliency detection models based on multi-task learning techniques $[24,25]$. Seo et al. introduced the concept of self-resemblance to measure visual saliency for video frames [36].

Most of the above saliency detection models estimate the saliency map for images/video based on the center-surround difference calculation and there are some differences among them. The first difference is how to extract the image features to calculate the center-surround differences. Existing studies compute the center-surround differences for saliency estimation based on the low-level features directly $[11,16]$, features in transform domain $[10,12]$, etc. The second difference is how much of the surrounding region should 
be selected to calculate the center-surround differences for the center region. The studies in $[11,16,30]$ use the closest surrounding regions of the center region to calculate the center-surround differences. These methods mainly considers the local contrast for saliency detection. On the contrary, some other studies [7, 42] use the surrounding regions of the center patch including all the other patches in the input image for center-surround difference calculation. In those studies, they mainly focus on the global contrast for the saliency detection. The final difference is how to weight the center-surround difference for the saliency calculation. Some studies utilize the Euclidean distances between the center region and the surrounding regions to weight the center-surround differences [7], while others use the Gaussian distribution of the Euclidean distances between the center region and the surrounding regions [42]. However, in these existing studies, both the choices of surrounding regions and the weighting of the center-surround differences are not justified from the aspect of theories of the HVS.

In this study, we build a new acuity-inspired saliency detection model by using the human visual acuity and sparse features. The sparse features of image patches are used to calculate the center-surround differences. Different from existing studies, the human visual acuity is adopted to weight the center-surround differences for the saliency calculation. Furthermore, we use the characteristics of the HVS to choose the neighboring image patches (surrounding regions) for the saliency calculation of each image patch. Additionally, the center-bias factor is integrated into the proposed model to enhance the saliency map. Experimental results demonstrate the superior performance of the proposed saliency detection model over other existing 
ones on a large public image database with ground truth.

\section{Proposed Model}

The framework of the proposed model is depicted in Figure 1. First, the input image is divided into patches and the sparse features are extracted from each image patch. The center-surround differences between image patches are then calculated based on the sparse features. Meanwhile, the human visual acuity is computed by the spatial distance between image patches. The saliency map is computed from the center-surround differences weighted by visual acuity. We also adopt the center-bias factor to enhance the saliency map.

\subsection{Sparse Feature Extraction}

Previous studies have shown that the receptive field properties of simple cells in the primary visual cortex account for a sparse coding $[8,35]$. The images can be represented by a linear superposition of a set basis functions and the basis functions are adopted to maximize the sparsity of the image representation. These basis functions are obtained through training a large number of images and they strongly resemble the receptive field properties found in the primary visual cortex. Given an image $I$, its sparse features $f_{i}(i \in\{1,2, \ldots, n\})$ can be described as its response to the bank of filter functions $W_{i}(i \in\{1,2, \ldots, n\})$.

$$
f_{i}=W_{i} I
$$

The sparse coding strategy tries to find an optimal weighting matrix $W$ such that the image $I$ can be represented by sparse features. Recently, many 
studies have focused on improving the efficiency of sparse coding $[9,20]$ or optimization of the structured sparsity [33, 37]. In [20], Lee et al. presented efficient sparse coding algorithms by iteratively solving L1-regularized least squares and L2-constrained least squares problems. Gregor et al. proposed to train a non-linear, feed-forward predictor in a specific architecture and a fixed depth to obtain the optimal approximation of the sparse code efficiently [9]. In [37], Stojnic et al. investigated the recovery of block sparse signals based on an under-determined set of equations from random Gaussian matrices. Mairal et al. introduced new network flow optimization algorithms for structured sparsity [33]. Some other studies investigate the applications of sparse coding in image restoration [32], traffic sign recognition [26], face recognition [44], etc.

Similar with sparse coding, ICA (Independent Component Analysis) [2, 13] can be used to extract the sparse features for images. Based on the linear transform of images, the ICA algorithms decompose images into independent components. With ICA, an image can be represented as a linear combination of a set of independent components. An alternative of Eq. (1) to represent an image $I$ by ICA can be expressed as:

$$
I=\sum_{i} f_{i} A_{i}
$$

where $f_{i}$ is the sparse features of the image, representing the coefficients of the independent components $A_{i}$ for the image $I$; and $W_{i}=A_{i}^{-1}$.

At present, many ICA algorithms have been proposed for feature extraction of visual signals $[2,13]$. In this study, we use the robust ICA algorithm in [13] to learn a set of independent components for natural image patches. These components are used to generate the sparse features of image patches 
in the proposed saliency detection model. We use over 180,000 image patches with the size of $8 \times 8 \times 3$ randomly extracted from natural scenes to learn a set of 192 independent components. The first 64 components $A$ and their corresponding filter functions $W$ are shown in Figure 2 (a) and (b), respectively.

\subsection{Human Visual Acuity}

The human visual acuity can be measured by a function of the retinal eccentricity, and to be more specific, the contrast threshold can be represented as a function of the spatial frequency and retinal eccentricity as follows.

$$
T(f, e)=T_{0} \exp \left(\alpha f \frac{e+e_{2}}{e_{2}}\right)
$$

where $f$ is the spatial frequency (cycles/degree); $e$ is the retinal eccentricity (degrees); $T_{0}$ is the minimum contrast threshold; $\alpha$ is the spatial frequency decay constant and $e_{2}$ is the half-resolution eccentricity. According to the experimental results in [6], these parameters are set to $T_{0}=1 / 64, \alpha=0.106$ and $e_{2}=2.3$. The human visual acuity can be represented as the reciprocal of the contrast threshold as follows.

$$
C(f, e)=1 / T(f, e)
$$

Generally, the typical viewing distance is 3 to 6 times of the image height [17]. We set the viewing distance as 4 times of the image height to calculate the human visual acuity based on Eqs. (3) and (4). The human visual acuity is used as the weighting for the center-surround differences between image patches in the proposed model. The advantage of this weighting will be illustrated in the next subsection. 


\subsection{Saliency Detection}

\subsubsection{Saliency Estimation for Each Image Patch}

In this study, the saliency value of each image patch is determined by the weighted patch differences from the neighboring image patches. Given an image patch $p$ in the input image, its saliency is calculated as:

$$
S_{p}=\sum_{q \neq p} C\left(f, e_{p q}\right) D_{p q}
$$

where $e_{p q}$ is the retinal eccentricity of the image patch $q$ from the fixation image patch $p$, and $D_{p q}$ is the patch difference between image patches $p$ and $q$.

Here, we use the human visual acuity as the weighting of the patch differences to calculate the final saliency map. From Eqs. (3) and (4), we can see that the human visual acuity decreases with larger eccentricity from the center patch, and this means that the contribution of the center-surround difference from the farther image patch is less than that from the nearer image patch from the center patch. This is reasonable and consistent with the characteristics of the HVS, for the center-surround difference from the farther image patch has less influence to the saliency value of the center patch, compared with the nearer image patch of the center patch.

The human visual acuity $C_{\left(f, e_{p q}\right)}$ can be obtained by Eq. (4) and it is used as the weighting of the center-surround difference $D_{p q}$ between image patches $p$ and $q$. We compute $D_{p q}$ based on sparse features $f_{i}$ as follows.

$$
D_{p q}=\sum_{i}\left|f_{i}(p)-f_{i}(q)\right|
$$

where $f_{i}(p)$ and $f_{i}(q)(i \in 0,1,2, \ldots, n)$ represent the sparse features of image patches $p$ and $q$, respectively; |.| is L2 norm. 


\subsubsection{Neighboring Image Patch Choice}

As introduced above, the saliency value of each image patch is calculated based on the patch differences from the neighboring image patches. In [16], the authors use a small part of near neighboring pixels to calculate the saliency value of the center pixel. In [7, 27], all the neighboring patches of the center image patch are involved to calculate the center-surround differences for saliency computation of each center patch; compared with Itti's model [16] which only takes the local contrast into consideration, these studies combine local and global contrast for saliency detection.

In this study, we select the neighboring image patches of each center image patch according to the characteristics of the HVS. Previous studies have shown that the retinal area within 1 to 2 degrees in the fovea is with the best visual acuity and the parafovea around the fovea is with low visual acuity [19]. Here, the retinal eccentricity $e_{0}$ is used to represent the area with the best visual acuity, which is set as 2 degrees. The neighboring patches of each center patch should be within the area with the retinal eccentricity $e_{0}$. We calculate the maximum distance $d_{m}$ between the center image patch and the neighboring image patches within the area with the retinal eccentricity $e_{0}$. When computing the saliency value of the center patch, we choose those neighboring patches whose Euclidian distances are smaller than $d_{m}$.

\subsection{Center-Bias Enhancement}

Eye tracking experiments have demonstrated that the bias towards the screen center exists during human fixation, which is called center bias [38, 40]. The experiments in [38] show that the initial response is to orient to the screen

center when the scene appears. The center-bias has been also found during 
human fixation [40]. In this study, we use the center-bias factor to enhance the saliency map for the input image. Similar with the study [38], we use a Gaussian function with kernel width as one degree (foveal size) to model the center bias factor. The center bias map $(\mathrm{CBM}) S_{c}$ is obtained by the Gaussian function.

Experimental results in the study [38] indicate that the center bias is irrespective to the distortion of image features, which demonstrates that the center bias is independent to the saliency map $S_{f}$ calculated from image features. Here, we use the CBM $S_{c}$ to represent the fixation map from the center bias factor, independent to the fixation map $S_{f}$ estimated from image features. The final saliency map by considering the center bias factor is calculated as follows.

$$
S=\gamma_{1} S_{f}+\gamma_{2} S_{c}+\gamma_{3} S_{f} * S_{c}
$$

where $\gamma_{1}, \gamma_{2}$, and $\gamma_{3}$ are parameters used to determine the weighting of each component.

\section{Experimental Results}

In this section, we conduct the comparison experiments to demonstrate the performance of the proposed model by using the public database [1]. This database includes 1000 original images and the corresponding ground-truth of accurately human-labeled salient objects. Eight relevant existing saliency detection models are used in the comparison experiments: CA [7], FT [1], MZ [30], GB [11], MR [10], SR [12], IT [16] and NL [34]. The reason we choose these saliency detection models for comparison is as follows: IT is a 
early saliency detection model designed based on the biological theory, while GB is an improved version on IT; MZ is a early saliency detection model which are built in the spatial domain; SR, MR and NL are built on other transform domains (SR and MR are implemented in the Fourier Transform, while NL is implemented in the Wavelet Transform.); in addition, similar with the saliency detection models of CA, MZ, IT and GB, the proposed saliency detection model is also devised based on the center-surround differences.

\subsection{Quantitative Evaluation}

To compare these saliency detection models quantitatively, we use three common measures in the comparison experiments: Pearson Linear Correlation Coefficient (PLCC) [23], Kullback-Leibler Divergence (KLD) [3, 23], and Receiver Operating Characteristic (ROC) [7, 14]. PLCC and KLD are calculated directly from the comparison between the ground truth map labelled by observers and the saliency map predicted from the saliency detection model. With a larger PLCC value, the saliency detection model can predict more accurate salient regions for images. In contrast, the performance of the saliency detection model with a smaller KLD value computed from the ground truth and saliency map is better than that of the saliency detection models with larger KLD values. ROC is a widely used measure for performance evaluation of saliency detection models $[7,14]$. Through defining a threshold, the saliency map can be divided into the salient points and non-salient points. The ground-truth map is marked as target points or background points by subjects. The percentage of the target points falling into the salient points in a saliency map is True Positive Rate (TPR), while the percentage of background points falling into the salient points is False Positive Rate (FPR). 
Increasing the threshold will result in less salient points, corresponding to the smaller TPR and FPR. The ROC curve of a specific saliency detection algorithm is a graphical plot of TPR VS. FPR with varied thresholds.

PLCC and KLD values of the compared saliency detection models are shown in Table. 1. From this table, we can see that CA and GB can get higher PLCC and lower KLD values than other existing saliency detection models, which means that $\mathrm{CA}$ and GB can predict more accurate saliency results than other existing ones. These experimental results are coincident with those reported in the study [7]. Among all the compared models, the proposed model can obtain the best prediction performance for saliency detection, as shown by PLCC and KLD values in Table 1. The paired t-test for the performance comparison is also conducted and the experimental results are shown in Table 1. From this table, we can see that the performance of the proposed model is significantly better than that of existing ones. The ROC curves in Figure 3 also demonstrate that the proposed model can obtain better performance of saliency estimation than other existing ones. In summary, according to the experimental results shown in Figure 3 and Table 1 , the overall performance of the proposed saliency detection model is significantly better than that of other existing ones.

\subsection{Examples for Visualization and Further Discussion}

We also provide some visual comparison samples in Figures 4 and 5. From Figure 4, we can see that MZ mainly detects the contour of the salient object in the saliency map, since it estimates the saliency map for images based on local contrast. For MR, it cannot detect the complete salient regions in the image, since it calculates the saliency map for images based on global 
contrast. Although CA considers both global and local contrast, it still suffers the similar problem (i.e., it just detects the contour of the salient objects and thus lose much salient information), as shown in the second column of Figure 4. Compared with MZ, MR and CA, FT estimates the saliency map for images with more low-frequency information. However, they would wrongly detect some background information as the salient regions (such as the saliency maps from the first, second and third images in Figure 4) or lose some salient information (such as the saliency map from the fifth image in Figure 4). Compared with other existing models, the proposed model can obtain much better saliency results, as shown in the six column of Figure 4.

From Figure 5, we can see that SR suffers the same problem with MR for their similar methodology in saliency estimation. GB and IT calculate the saliency map based on local contrast and thus they would mainly detect the contour of salient objects, as shown in the second and fourth columns in Figure 5. NL wrongly detects much background region as the salient region, as shown in fifth column of Figure 5. Compared with these existing models, the proposed model can predict more accurate saliency results, as demonstrated in the six column of Figure 5.

\subsection{Additional Analysis Related to Parameter Settings}

In this experiment, we evaluate the performance of the proposed model with different parameter settings in Eq. (7). From Eq. (7), we can see that the final saliency map can be calculated by different fusion methods with different parameter settings. Here, we use different parameter settings in Eq. (7) to obtain different saliency detection models to conduct the comparison experiments as follows: (1) CBM: $\gamma_{1}=0, \gamma_{2}=1$, and $\gamma_{3}=0$. In this 
case, the final saliency map is the CBM $S_{c}$. (2) Proposed Model w/o CBM: $\gamma_{1}=1, \gamma_{2}=0$, and $\gamma_{3}=0$. In this case, the final saliency map is the predicted saliency map $S_{f}$ from the saliency detection model. (3) SUM: $\gamma_{1}=1, \gamma_{2}=1$, and $\gamma_{3}=0$. In this case, the final saliency map is the sum of the CBM $S_{c}$ and predicted saliency map $S_{f}$. (4) MULT: $\gamma_{1}=0, \gamma_{2}=0$, and $\gamma_{3}=1$. In this case, the final saliency map is the multiplication of the CBM $S_{C}$ and predicted saliency map $S_{f}$. (5) Proposed Model: $\gamma_{1}=1, \gamma_{2}=1$, and $\gamma_{3}=1$. In this case, the final saliency map is the combination of SUM and MULT.

We provide the experimental results of the proposed model with different parameter settings in Table 2 and Figure 6. From Table 2, we can see that SUM (the sum of the CBM $S_{c}$ and predicted saliency map $S_{f}$ ) can obtain better performance than the CBM or Proposed Model w/o CBM, which demonstrates that the CBM can be used to enhance the saliency results. For MULT, its performance is lower than that of the Proposed Model w/o CBM, which indicates that the fusion method by multiplication can not work well. From the last column in Table 2, the proposed model with $\gamma_{1}=1, \gamma_{2}=1$, and $\gamma_{3}=1$ in Eq. (7) obtains the best performance among these models with different parameter settings. The ROC curves in Figure 6 also show the similar results.

\section{Conclusion}

In this paper, we have proposed a human-acuity based saliency detection model. The proposed model calculates the saliency map of images based on two characteristics relevant to the HVS: the human visual acuity and 
sparse features. The sparse features of image patches extracted based on the learned independent components are used to calculate the center-surround differences between image patches, while the human visual acuity is utilized to weight these patch differences. We also use the characteristics of the HVS to select the neighboring image patches for the saliency calculation of the center image patch. Additionally, the center-bias factor is adopted to enhance the saliency map. Compared with other existing models, experimental results on a large public database show the better performance of the proposed saliency detection model.

\section{Acknowledgement}

We are grateful for the editor and anonymous reviewers' insightful comments that have prompted us for re-thinking and re-positioning of our work. This work was partially supported by the Funds from SRF for ROCS, SEM, DoE of Jiangxi Province (No.GJJ14347), NSF of Jiangxi Province (No. 20142 BAB217011), and NSF of China (No.61461021, 61363038).

\section{References}

[1] R. Achanta, S. Hemami, F. Estrada and S. Susstrunk. Frequency-tuned salient region detection. IEEE International Conference on Computer Vision and Pattern Recognition, 2009.

[2] A. J. Bell and T. J. Sejnowski. The independent components of natural scenes are edge filters. Visual Research, 37: 3327-3338, 1997.

[3] N. Bruce, and J. Tsotsos. Saliency, attention and visual search: an information theoretic approach. Journal of Vision, 9(3), 2009. 
[4] Y. Fang, W. Lin, C. T. Lau, B.-S. Lee. A visual attention model combining top-down and bottom-up mechanisms for salient object detection. IEEE International Conference on Acoustics, Speech, and Signal Processing, 2011.

[5] D. Gao and N. Vasconcelos. Bottom-up saliency is a discriminant process. IEEE International Conference on Computer Vision, 2007.

[6] W. S. Geisler and J. S. Perry. A real-time foveated multi-solution system for low-bandwidth video communication. In Proc. of SPIE, 3299: 294305, 1998.

[7] S. Goferman, L. Zelnik-Manor, and A. Tal. Context-aware saliency detection. IEEE International Conference on Computer Vision and Pattern Recognition, 2010.

[8] D. Graham and D. J. Field. Efficient coding of natural images. New Encyclopedia of Neuroscience, 2007.

[9] K. Gregor and Y. LeCun. Learning fast approximations of sparse coding. International Conference on Machine Learning, 2010.

[10] C. Guo, Q. Ma and L. Zhang. Spatio-temporal saliency detection using phase spectrum of quaternion fourier transform. IEEE International Conference on Computer Vision and Pattern Recognition, 2008.

[11] J. Harel, C. Koch and P. Perona. Graph-based visual saliency. Proceedings of the Neural Information Processing Systems (NIPS), 2006. 
[12] X. Hou and L. Zhang. Saliency detection: a spectral residual approach. IEEE International Conference on Computer Vision and Pattern Recognition, 2007.

[13] A. Hyvarinen. Fast and robust fixed-point algorithms for independent component analysis. IEEE Transactions on Neural Network, 10(3): 626634, 1999.

[14] N. Imamoglu, W. Lin, and Y. Fang. A Saliency Detection Model Using Low-Level Features Based on Wavelet Transform. IEEE Transactions on Multimedia, 15(1): 96-105, 2013.

[15] L. Itti, and P. Baldi. Bayesian Surprise Attracts Human Attention. Advances in Neural Information Processing Systems, 2006.

[16] L. Itti, C. Koch and E. Niebur. A model of saliency-based visual attention for rapid scene analysis. IEEE Transactions on Pattern Analysis and Machine Intelligence, 20(11): 1254-1259, 1998.

[17] ITU. Methodology for the subjective assessment of the quality of television pictures. Geneva, Switzerland, ITU-R BT.500-11, 2002.

[18] M. Jian, K.-M. Lam, J. Dong. Facial-feature detection and localization based on a hierarchical scheme. Information Sciences, 262: 1-14, 2014.

[19] M. A. Just and P. A. Carpenter. The psychology of reading and language comprehension. Newton, MA: Allyn and Bacon, 1987.

[20] H. Lee, A. Battle, R. Raina, and A. Y. Ng. Efficient sparse coding 
algorithms. Proceedings of the Neural Information Processing Systems (NIPS), 2006.

[21] J. Lei, H. Zhang, L. You, C. Hou, and L. Wang. Evaluation and modeling of depth feature incorporated visual attention for salient object segmentation. Neurocomputing, vol.120: 24-33, 2013.

[22] O. Le Meur, P. Le Callet and D. Barba. Predicting visual fixations on video based on low-level visual features. Vision Research, 47(19): 24832498, 2007.

[23] O. Le Meur, P. Le Callet, D. Barba, and D. Thoreau. A coherent computational approach to model bottom-up visual attention. IEEE Transactions on Pattern Analysis and Machine Intelligence, 28(5): 802-817, 2006.

[24] J. Li, Y. Tian, T. Huang and W. Gao. Probabilistic Multi-Task Learning for Visual Saliency Estimation in Video. Intl J. Comput. Vision, 90(2): 150-165, 2010.

[25] J. Li, Y. Tian, T. Huang and W. Gao. Multi-Task Rank Learning for Visual Saliency Estimation. IEEE Transactions on Circuits and Systen for Video Technology, 21(5): 623-636, 2011.

[26] H. Liu, Y. Liu and F. Sun. Traffic sign recognition using group sparse coding. Information Sciences, 266: 75-89, 2014.

[27] T. Liu, J. Sun, N. Zheng, X. Tang and H. Y. Shum. Learning to detect a salient object. IEEE International Conference on Computer Vision and Pattern Recognition, 2007. 
[28] Z. Lu, W. Lin, X. Yang, E. Ong and S. Yao. Modeling visual attention's modulatory aftereffects on visual sensitivity and quality evaluation. IEEE Transactions on Image Processing,14 (11): 1928 - 1942, 2005.

[29] Y. Ma, X. Hua, L. Lu, and H. Zhang. A Generic framework of user attention model and its application in video summarization. IEEE Transactions on Multimedia, 7 (5): 907-919, 2005.

[30] Y. Ma and H. Zhang. Contrast-based image attention analysis by using fuzzy growing. ACM International Conference on Multimedia, 2003.

[31] V. Mahadevan and N. Vasconcelos. Spatiotemporal saliency in dynamic scenes. IEEE Transactions on Pattern Analysis and Machine Intelligence, 32(1): 171-177, 2010.

[32] J. Mairal, M. Elad and G. Sapiro. Sparse representation for color image restoration. IEEE Transactions on Image Processing, 17(1):53-69. 2008.

[33] J. Mairal, R. Jenatton, G. Obozinski, and F. Bach. Convex and network flow optimization for structural sparsity. Journal of Machine Learning Research, 12: 2681-2720, 2011.

[34] N. Murray, M. Vanrell, X. Otazu, and C. A. Parraga. Saliency estimation using a non-parametric low-level vision model. IEEE International Conference on Computer Vision and Pattern Recognition, 2011.

[35] B. A. Olshausen and D. J. Field. Emergence of simple-cell receptive field properties by learning a sparse code for natural images. Nature, 381: 607-609, 1996. 
[36] H. J. Seo, and P. Milanfar. Static and Space-time Visual Saliency Detection by Self-Resemblance. The Journal of Vision, 9(12):15, 1-27, 2009.

[37] M. Stojnic, F. Parvaresh, and B. Hassibi. On the reconstruction of block-sparse signals with an optimal number of measurements. IEEE Transcations on Signal Processing, 57(8): 3075-3085, 2009.

[38] B. W. Tatler. The central fixation bias in scene viewing: Selecting an optimal viewing position independently of motor biases and image feature distributions, Journal of Vision, 7(14):4, 1-17, 2007.

[39] A. Treisman and G. Gelade, A feature-integration theory of attention. Cognitive Psychology, 12 (1): 97-136, 1980.

[40] P. Tseng, R. Carmi, I. Cameron, D. Munoz, and L. Itti. Quantifying center bias of observers in free viewing of dynamic natural scenes. Journal of Vision, 9(7):4, 1-16, 2009.

[41] B. A. Wandell. Foundations of Vision. newblock Sunderland, MA: Sinauer Associates, Inc, 1995.

[42] W. Wang, Y. Wang, Q. Huang, and W. Gao. Measuring visual saliency by site entropy rate. IEEE International Conference on Computer Vision and Pattern Recognition, 2010.

[43] J. M. Wolfe, S. J. Butcher and M. Hyle, Changing your mind: On the contributions of top-down and bottom-up guidance in visual search for feature singletons. Journal of Experimental Psychology: Human Perception and Performance, 29 (2): 483-502, 2003. 
[44] M. Yang, L. Zhang, J. Yang and L. Zhang. Robust sparse coding for face recognition. IEEE International Conference on Computer Vision and Pattern Recognition, 2011.

[45] Y. Zhai, and M. Shah. Visual attention detection in video sequences using spatiotemporal cues. ACM international conference on multimedia, 2006 .

[46] Y. Zhang, Z. Mao, J. Li, and Q. Tian. Saliency region detection for complex background images using integrated features. Information Sciences, 281: 586-600, 2014. 


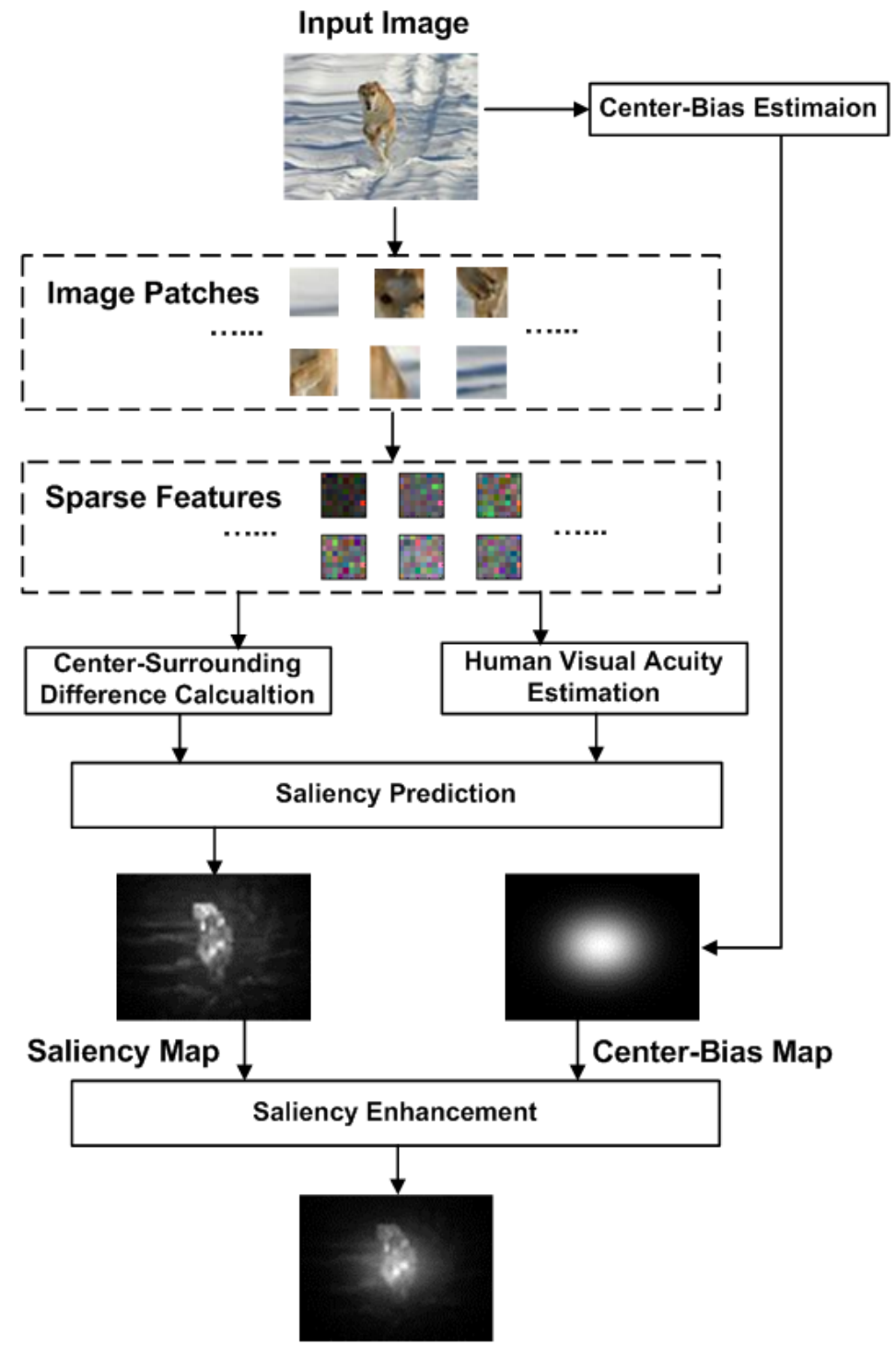

Final Saliency Map

Figure 1: The proposed framework. 


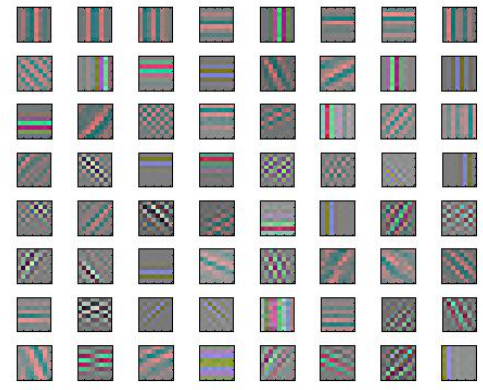

(a)

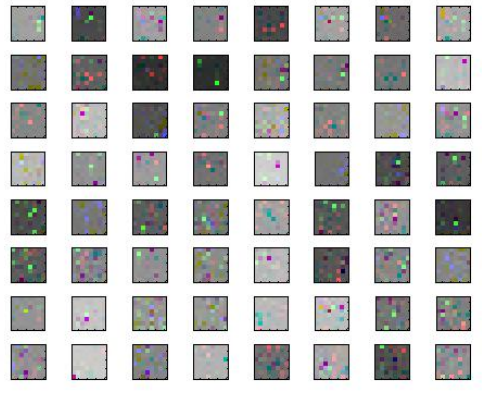

(b)

Figure 2: (a) The first 64 sparse basis $A$ learned from the natural image patches. (b) The corresponding first 64 filter functions $W$ of the sparse basis $A$.

Table 1: Comparison of different saliency detection models by KLD and PLCC. * means that the performance of the compared model is significantly different from that of the proposed model (paired t-test, $p<0.05$ ).

\begin{tabular}{|c|c|c|c|c|c|c|c|c|c|}
\hline Models & CA & FT & MZ & GB & MR & SR & IT & NL & $\begin{array}{c}\text { Proposed } \\
\text { Model }\end{array}$ \\
\hline \hline KLD & $1.057^{*}$ & $1.335^{*}$ & $1.370^{*}$ & $1.111^{*}$ & $1.124^{*}$ & $1.401^{*}$ & $1.363^{*}$ & $1.422^{*}$ & $0.987^{*}$ \\
\hline \hline PLCC & $0.558^{*}$ & $0.496^{*}$ & $0.375^{*}$ & $0.549^{*}$ & $0.519^{*}$ & $0.364^{*}$ & $0.357^{*}$ & $0.379^{*}$ & $0.579^{*}$ \\
\hline
\end{tabular}

Table 2: Comparison of the proposed saliency detection model with different parameter settings.

\begin{tabular}{|c|c|c|c|c|c|}
\hline Models & CBM & $\begin{array}{c}\text { Proposed Model } \\
\text { w/o CBM }\end{array}$ & SUM & MULT & Proposed Model \\
\hline \hline KLD & 1.554 & 1.032 & 0.995 & 1.662 & 0.987 \\
\hline \hline PLCC & 0.487 & 0.570 & 0.575 & 0.507 & 0.579 \\
\hline
\end{tabular}




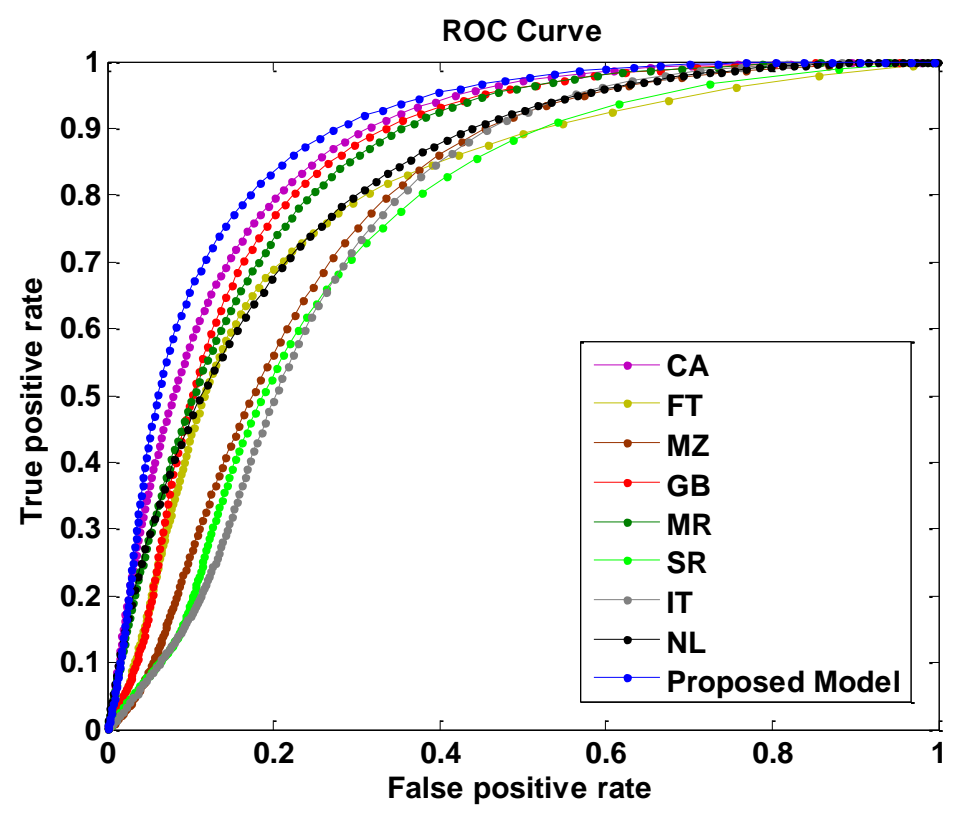

Figure 3: ROC Curves for different saliency detection models. 

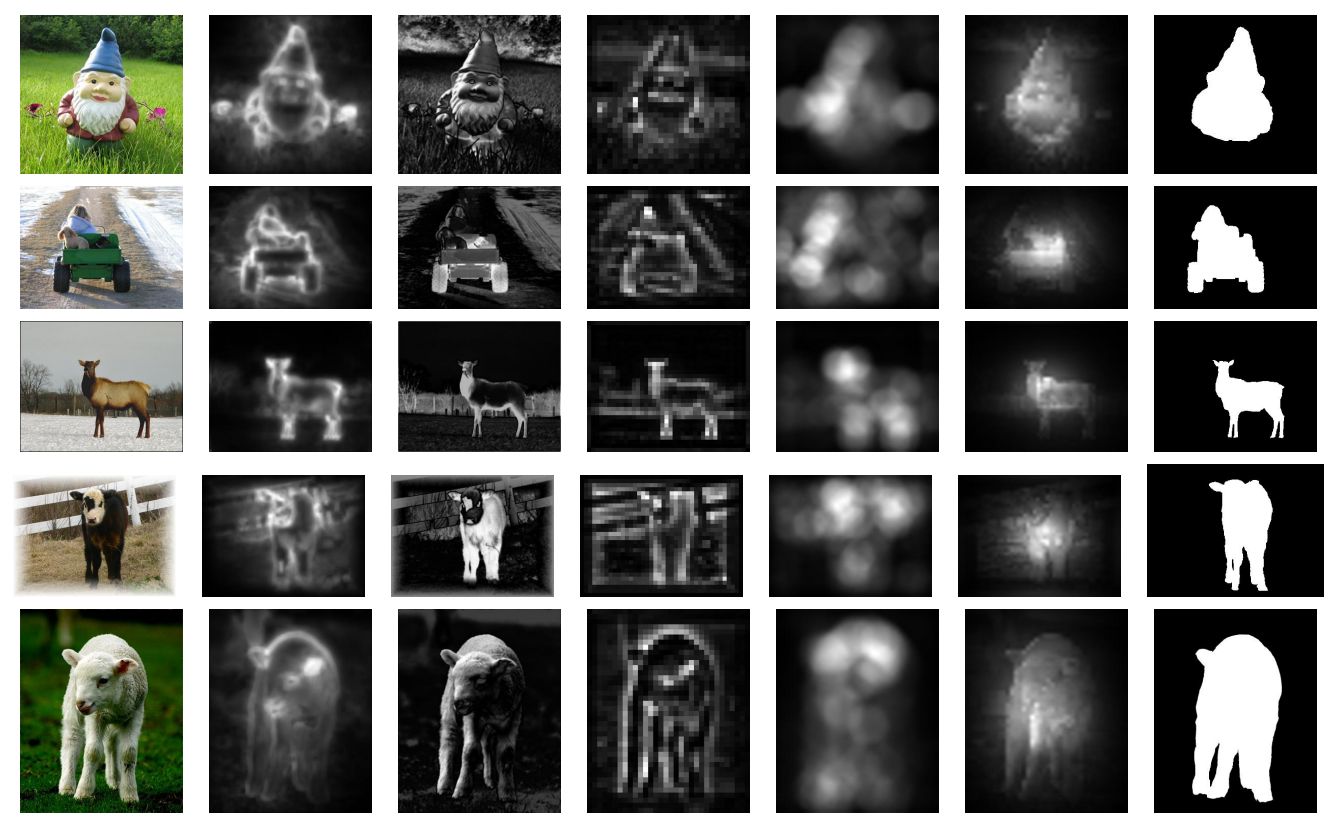

Figure 4: Comparison of different saliency detection algorithms: first column to the final column: original images, saliency maps from CA, FT, MZ, MR, the proposed model, and the ground truth, respectively. 


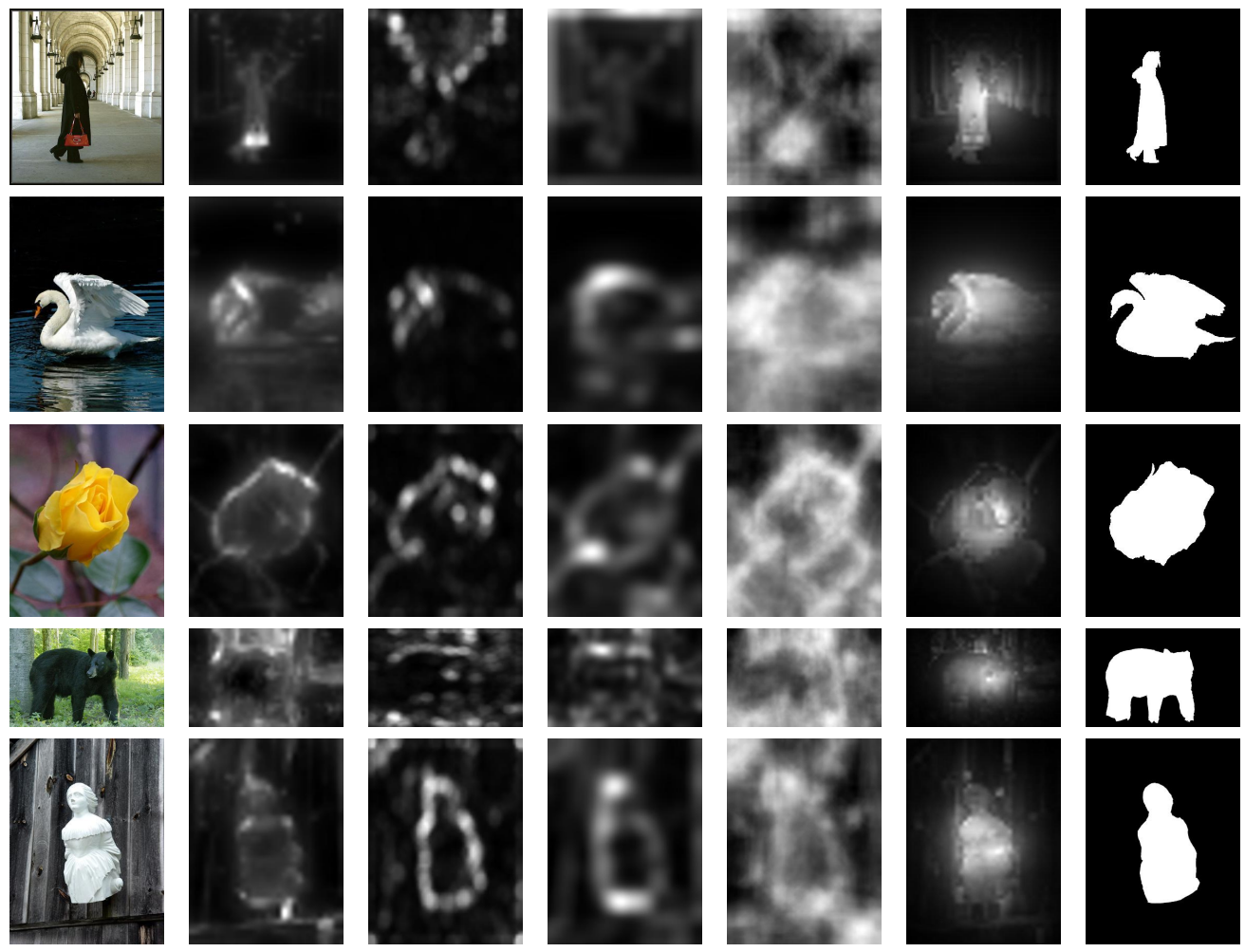

Figure 5: Comparison of different saliency detection algorithms: first column to the final column: original images, saliency maps from GB, SR, IT, NL, the proposed model, and the ground truth, respectively. 


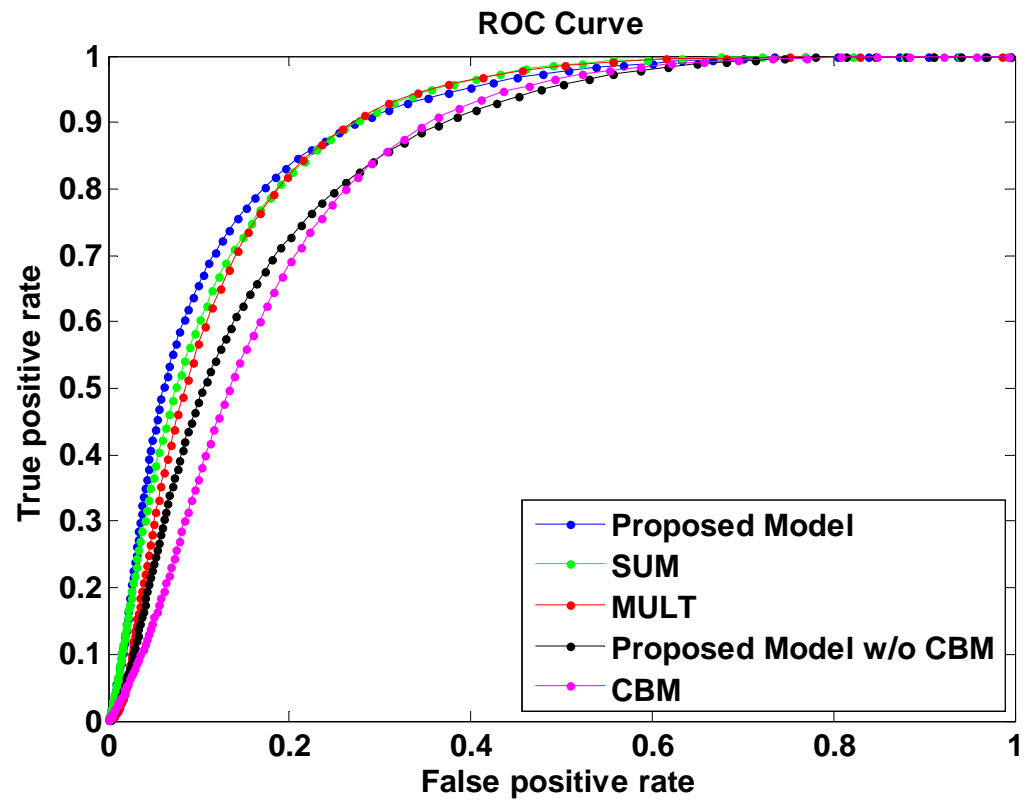

Figure 6: ROC Curves of the proposed saliency detection models with different different parameter settings. 